\title{
ANÁLISIS DE LA MOTIVACIÓN EN UN CONTEXTO 2.0 DE TRABAJO COLABORATIVO
}

\author{
Alfredo Álvarez-Álvarez¹: Universidad de Alcalá. España \\ a.alvarezalvarez@uah.es
}

\section{RESUMEN}

Se denomina ya con naturalidad a los jóvenes actuales como "nativos digitales". Asistimos a las primeras generaciones de ciudadanos que tienen en la electrónica y en la informática un recurso permanente en su devenir cotidiano. Sin embargo, el aula parece ser, en muchos sentidos, reducto de siglos pasados. El uso del ordenador en la clase no está generalizado y la utilización de las tecnologías se reduce, con frecuencia, a utilizar una plataforma CMS para dejar o consultar documentación, sin que se observen muchas posibilidades de interacción; el teléfono móvil está infrautilizado y las redes sociales están dando sus primeros pasos de manera aún un tanto dubitativa en el ámbito estrictamente académico. Sin embargo, la llamada web 2.0 ofrece en estos momentos, entre sus aportaciones más relevantes, una aproximación a la motivación distinta de lo que se ha conocido, especialmente en el aprendizaje de un idioma. En este contexto, se presentan los resultados de un estudio, con dos grupos de estudiantes, en los que se analizó la motivación, mediante encuestas y a través de los mecanismos que ofrece la red social utilizada para seguir la asignatura.

PALABRAS CLAVE: Redes sociales - motivación - aprendizaje de idiomas-lenguaje

\section{ANALYSIS OF MOTIVATION IN A WEB 2.0 COLLABORATIVE WORK ENVIRONMENT}

\begin{abstract}
\footnotetext{
${ }^{1}$ Autor correspondiente

Alfredo Álvarez-Álvarez: Profesor de la Universidad de Alcalá, Madrid, España

Correo: a.alvarezalvarez@uah.es
}

Today's youngsters are now dubbed "digital natives" as the first ever generation who have grown up alongside electronic and computer devices as part of their natural lifestyle. The classroom, on the other hand, seems in many ways to have been relegated to a relic of the past. Classroom computer use is not yet widespread and the use of technologies is often limited to use of the CMS for making enquiries and 
reference work without many interaction possibilities. The mobile phone is underused and social networking sites are still taking their nursery steps in the strictly academic world. Nonetheless, one of the most important inputs of the web 2.0 is a totally different motivation approach in language learning. This article presents the results of a motivation study involving two student groups using surveys and social networking resources for taking the subject.

KEY WORDS: Social network - motivation - language learning-language

\section{INTRODUCCIÓN}

\subsection{Argumentando con la red social como uno de los posibles paradigmas para un entorno colaborativo}

Las redes sociales aparecen al menos en 2002 en forma de espacios web que impulsaban las redes llamadas de círculos de amigos en línea, cuando el término se empleaba para describir las relaciones en las comunidades virtuales, concepto que se popularizó en 2003, con sitios como MySpace ${ }^{2}$ o Xing ${ }^{3}$. En la actualidad hay más de 200 sitios de redes sociales, aunque Friendster ${ }^{4}$ ha sido uno de los que mejor han sabido emplear la técnica del círculo de amigos ${ }^{5}$.

De acuerdo con el planteamiento general, en estas comunidades un número inicial de participantes envía mensajes a miembros de su propia red social invitándoles a unirse al sitio. Los nuevos participantes repiten el proceso, creciendo el número total de miembros y los enlaces de la red. Los sitios ofrecen diversas características, como actualización automática de la libreta, perfiles visibles, capacidad para crear nuevos enlaces mediante servicios de presentación y otras maneras de conexión social en línea.

Alrededor de 2001 y 2002 surgen los primeros sitios que fomentan redes de amigos. Hacia 2003 se hacen populares con la aparición de sitios tales como Tribe 6 y MySpace. En cuanto a las herramientas que proporcionan en general las redes sociales en Internet son:

a) Actualización automática de la libreta de direcciones.

b) Perfiles visibles.

c) Capacidad de crear nuevos enlaces mediante servicios de presentación y otras maneras de conexión social en línea.

\footnotetext{
2 http://www.myspace.com/

${ }^{3}$ http://www.xing.com/

4 http://www.friendster.com/

5 Teoría de los Seis grados de separación, según la cual toda la gente del planeta está conectada a través de no más de seis personas [http://es.wikipedia.org/wiki/Seis_grados_de_separaci\%C3\%B3n ].

6 http:/ / www.tribe.net/welcome
} 
Aunque las orientaciones más habituales de las redes sociales son las que tienen que ver con el ocio o el networking, hay otros ámbitos como el de la enseñanza, en el que parecen tener un espacio de actividad bastante apropiado si bien la todavía deficiente formación de los docentes, así como el desconocimiento de sus posibilidades de uso en el aula, hacen de esta herramienta un elemento de alto poder motivador y de gran potencia comunicadora, que está llamada, en un futuro más bien cercano, a disponer de un puesto de relevancia en el contexto educativo.

Las redes sociales ofrecen al menos cuatro características:

a) Representan una propuesta de trabajo nueva en el aula.

b) Aportan elementos novedosos, e innovadores, en el proceso de comunicación con el estudiante.

c) La posibilidad de interactuar en común se sitúa en la base de la generación de conocimiento.

d) Los estudiantes encuentran en ellas muchas razones para su uso.

Además, presentan algunas funcionalidades básicas que son especialmente útiles en la enseñanza. Entre ellas, las siguientes:

a) Posibilidad de creación de grupos.

b) Posibilidad de comunicación multidireccional.

c) Desarrollo de la función "Comentarios".

d) Posibilidad de incorporar enlaces e incluso aplicaciones externas.

e) Utilización de foros o chats.

f) Amplia disponibilidad para incorporación de documentos audio o vídeo.

g) Mensajería instantánea.

Sin embargo, en este proceso de incorporación de las redes sociales al aula, el papel del docente se propone como especialmente relevante. La introducción de las tecnologías provoca la aparición de nuevos elementos que hacen que el conocimiento se centre en la red y, por consiguiente, los docentes dejen de ser meros transmisores de información. En la actualidad, se enfrentan al reto de encauzar a los alumnos en el desarrollo de competencias y estrategias para alcanzar los objetivos que plantea la educación en nuestro siglo.

Ello quiere decir que el profesor es quien encauza al alumno más que en ningún otro momento de la historia, convirtiéndose en coacher, que dirige el proceso de aprendizaje de los estudiantes. En definitiva, es coordinador y no controlador, y su función es facilitar la consecución por parte de los estudiantes de los objetivos de las acciones formativas (Cabedo, 2011).

En relación a los estudiantes y el uso de las tecnologías y, más en particular, de las redes sociales, la realidad actual muestra que éstas forman parte integrante de sus vidas, lo cual quiere decir que, desde la óptica del profesor, éste no necesita enseñar su funcionamiento, ni determinar funcionalidades, ni tampoco dedicar tiempo a iniciarlos en su uso, bastará con establecer objetivos y orientar el ritmo y el recorrido. 
El joven, en relación con la red, es completamente utilitarista; no olvidemos que esta es la fuente de información que le proporciona, por ejemplo, su música y sus películas. Además, la red es nada menos que el espacio que le permite mantenerse en contacto con sus amigos. En ella se produce también una interacción constante que combina lo síncrono con lo asíncrono (Dans, 2010: 131), una circunstancia que el docente puede aprovechar para dinamizar la actividad de su grupo.

Desde este punto de vista, la red se convierte en el espacio del grupo, que puede representar un acercamiento entre docente y alumno. Desde el momento en que el estudiante se identifica con ese espacio y lo hace suyo, se implicará más en las actividades propuestas por el profesor, lo cual convierte a esta herramienta en un lugar de aprendizaje y enriquecimiento mutuo. El profesor interviene, por tanto, colaborando en el aprendizaje con sus alumnos. Por su parte, la red le proporciona diversas sinergias que hacen de ella un lugar idóneo para la socialización y la colaboración en diversas direcciones, todas ellas enriquecedoras y a través de las cuales se fomenta, entre otros aspectos, la propia autonomía del estudiante.

En definitiva, el espacio de interacción que puede otorgar una red social en el aula debería ser aprovechado para un modelo de aprendizaje común, colaborativo, cooperativo e interdisciplinar por parte del alumnado y del docente/los docentes. Así, estamos de acuerdo con Artero Balaguer (2010) en que se podrían establecer ciertas pautas u objetivos didácticos con el uso de redes sociales en el aula:

a) "Espacio de interacción, individualidad y multiculturalidad. Es decir, como lugar para el conocimiento compartido y el aprendizaje colaborativo."

b) "Espacio de metacognición. A través de actividades o tareas que conlleven la autoevaluación o reflexión sobre el trabajo realizado".

c) Espacio argumentativo. Plantear foros o interacciones sobre temas donde dejan aflorar las estrategias comunicativas de cada alumno, sus estrategias de búsqueda, selección de información y razonamiento, pero también donde se motive su rapidez de respuesta, su interacción y su comunicación. Esta búsqueda de capacidad de respuesta forma parte de la misma concepción de red social.

d) "Espacio como herramienta metodológica para el profesor. Por una parte, para alimentar su propia formación desde la práctica y participación en proyectos. Por otra, como observador, seguidor, mediador del trabajo de cada alumno y de las relaciones entre ellos."

\section{METODOLOGÍA}

\subsection{Una propuesta en distintos niveles : Contexto y participantes}

El estudio que se presenta fue realizado con dos grupos diferentes y en contextos distintos. En el primer caso, se trataba de un grupo de la asignatura de Traducción Científico-Técnica (Universidad Autónoma de Madrid) en el curso 2008-2009. Los estudiantes pertenecían a la Licenciatura de Traducción e Interpretación y cursaban 
3ro y 4to. En el segundo caso, se trató de un grupo de 2do curso del Grado de Estudios Ingleses, en la asignatura de Francés III (Universidad de Alcalá).

En el primer caso se trataba de una asignatura en la cual la actividad principal era la traducción y en el segundo el aprendizaje de un idioma, en particular, el Francés. El hecho de tratarse de actividades se ha considerado que aporta elementos positivos en la comparación.

Se pasaron dos cuestionarios, uno en la primera semana y otro en la última. En la mayor parte de los casos los estudiantes tenían la opción de múltiple respuesta así como la posibilidad de formular observaciones.

Entre las preguntas, una relacionada con la propia valoración de las TIC, una vez concluido el curso, es la que presenta quizá una mayor separación en cuanto a los conceptos que interesan en este artículo [Con ellas se aprende más rápido/Con ellas se aprende mejor/Son más entretenidas], y que se puede ver en la tabla adjunta

Tabla 1. Valoración de las TIC

\begin{tabular}{lcc}
\hline & Traducción 2008 & Français en ligne \\
Con ellas se aprende más rápido & $66.6 \%$ & $38 \%$ \\
Con ellas se aprende mejor & $73.3 \%$ & $53 \%$ \\
Son más entretenidas & $60 \%$ & $76 \%$ \\
Son iguales que otros métodos & $0 \%$ & $0 \%$ \\
Entorpecen el aprendizaje & $0 \%$ & $0 \%$ \\
\hline
\end{tabular}

Como queda patente, se produce una clara diferencia de valoración entre ambos grupos. Mientras en el ámbito de la traducción se produce una alta valoración en los tres conceptos, que llega al máximo con un $73 \%$ en Con ellas se aprende mejor, el grupo de estudio del Francés como lengua instrumental parece otorgar una mayor trascendencia al concepto Son más entretenidas frente a los otros dos.

Se trabajó con la red Ning, una de cuyas ventajas es que permite decidir la distribución de los espacios y, además, ofrece la opción de decidir si un usuario puede inscribirse o no, como miembro, en la red. Esta circunstancia hace que ya de partida, la plataforma resulte más atractiva, además de que disponga de una interfaz más intuitiva, un espacio blog para cada uno de sus miembros..., etc. 


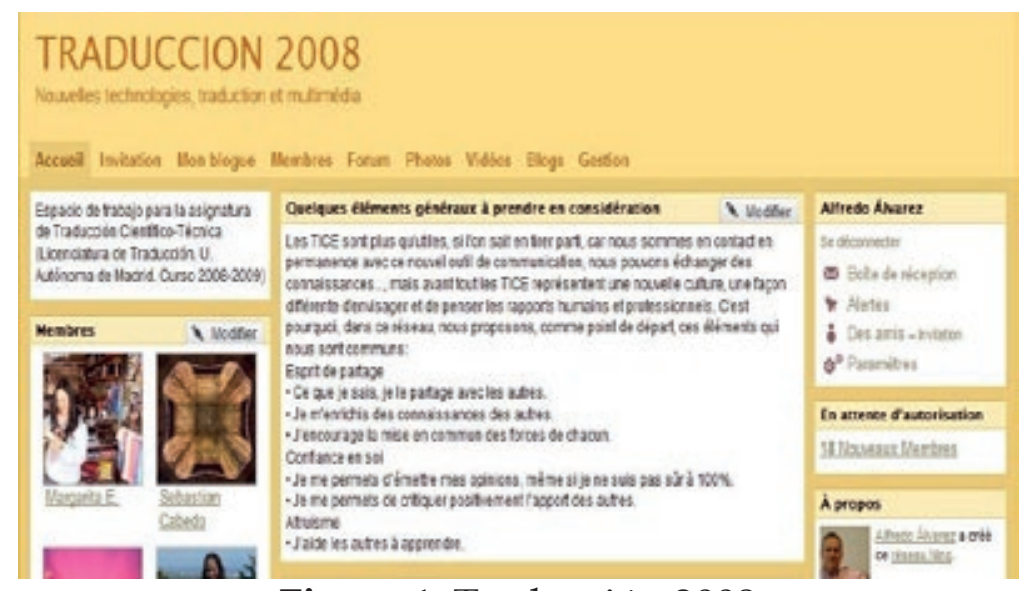

Figura 1. Traducción 2008

Tal como se ve en la Figura $n^{0} 1$, el espacio de la página de inicio se dividió en tres columnas, una primera en la que figuraba, arriba, la descripción del espacio de trabajo junto con las fotos de los participantes, fotos de cualquier otro tipo, documentos de vídeo o de música que cada cual quisiera aportar, y un último espacio destinado a convocatorias, que se abrió en los últimos días del semestre.

En la columna central, considerada la más visible por razones obvias, aparecen algunas indicaciones sobre el uso de la plataforma en primer lugar, las entradas de blog a continuación, un espacio destinado a referenciar las actividades más recientes y un foro de debate.

Finalmente, la tercera columna se destinó a la publicación de los textos para traducir, junto con otras informaciones, como el programa de la asignatura, tal como se puede ver en la Figura 2.

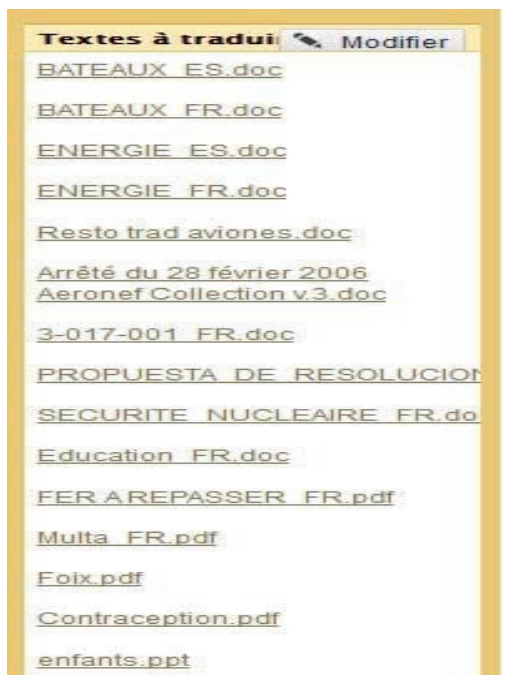

Figura 2. Programa de la asignatura

En cuanto a las actividades, éstas se dividieron en obligatorias y voluntarias. En el primer grupo se encontraban la traducción de cada texto propuesto por el profesor en su correspondiente espacio y un análisis de un recurso en línea. En concreto, un 
diccionario o banco de diccionarios, mediante una parrilla que se proporcionó a los alumnos junto con la distribución de cada recurso. Esta actividad incluía, además, una presentación oral.

En lo que se refiere a las actividades de carácter voluntario se intentó básicamente que el estudiante se posicionara frente a la realidad que le rodea. Para ello se le propuso reseñar en el foro tanto cuestiones de tipo terminológico, como la detección y comentario de aquellas expresiones, contrarias a la norma, que pudiera descubrir en los medios de comunicación especialmente, como transmisores que son tanto de la norma como de sus incumplimientos. El resultado arroja un total de 56 temas iniciados, a los que hay que añadir sus réplicas y contrarréplicas, todo lo cual asciende a 105 inserciones.

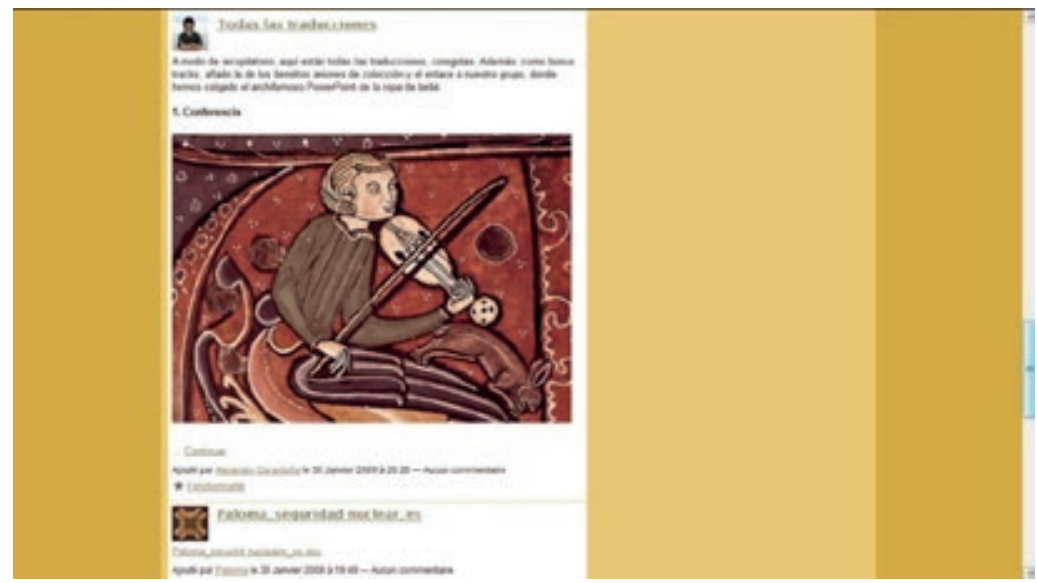

Figura 3. Ejemplo de réplica

Con anterioridad se ha mencionado el hecho de que, al hacer la inscripción en la red, Ning abre un espacio blog individual en el que quedan publicados los posts de cada miembro. Lo cual es de gran utilidad pedagógica ya que, cuando el profesor desea comprobar el trabajo realizado por un determinado estudiante, sólo tiene que pinchar en su foto, que le dará acceso al blog, en el que encontrará la información, junto con la fecha y hora de su publicación.

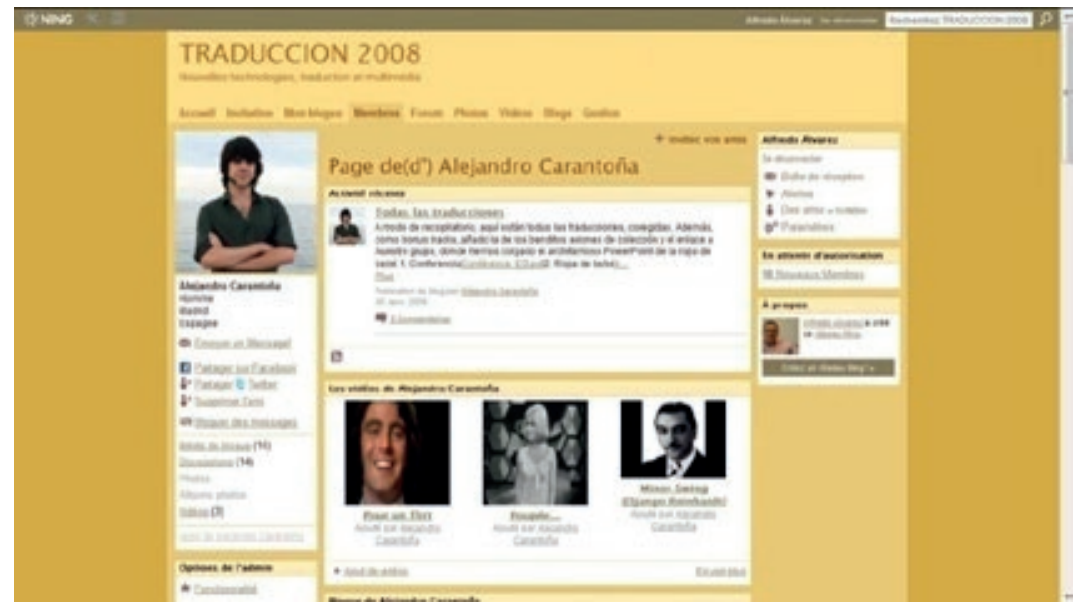

Figura 4. Los posts de Ning 
Ésta vendrá estructurada con el siguiente orden:
a) $3 / 4 \quad$ Actividad reciente
b) $3 / 4 \quad$ Fotos
c) $3 / 4 \quad$ Vídeos
d) $3 / 4 \quad$ Blog
e) $3 / 4 \quad$ Muro de comentarios.

Esta organización facilita enormemente la tarea de evaluación, así como la verificación de tareas propuestas para realizar en autonomía, al tiempo que asegura un mejor ordenamiento de los espacios.

En lo que respecta al segundo grupo, básicamente se organizó de la misma manera que Traducción 2008, en una distribución en tres columnas, de la forma que se presenta a continuación:

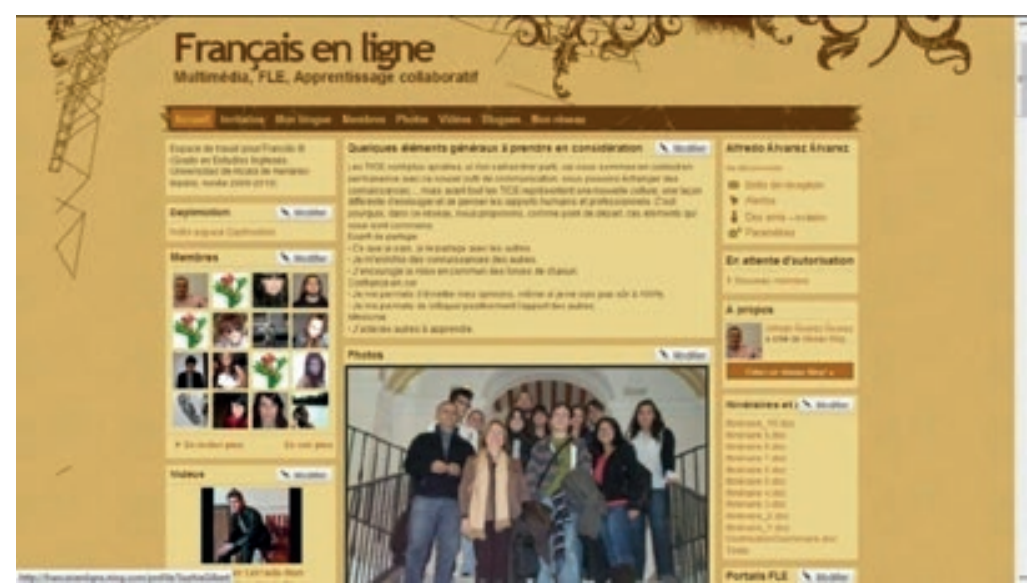

Figura 5. Los posts de Ning

La diferencia entre ambas, como se ha mencionado anteriormente, tiene relación con el tipo de actividades realizadas en un caso y en otro. Français en ligne es una red que tiene por objeto el aprendizaje instrumental de una lengua. Por tanto, a diferencia del primer grupo, en el cual la actividad principal era la traducción, en este caso las tareas a realizar no tenían como objetivo la publicación en exclusiva de trabajos realizados por escrito. Más en concreto, sus actividades en la red tenían relación con el desarrollo de tres de las competencias básicas en el aprendizaje de un idioma: comprensión oral (y audiovisual), comprensión y expresión escritas. Para ello, dichas actividades, hasta un total de 23 calificables en el semestre, fueron básicamente de tres tipos: producción de documentos audio, producción de documentos vídeo, producción de documentos escritos y trabajo (en presencial y/o en autonomía) con recursos de carácter autocorrectivo, mediante la realización de itinerarios propuestos por el profesor.

En el caso de los estudiantes que utilizaron Français en Ligne, el porcentaje de actividades realizadas efectivamente fue del $95,5 \%$ de las 23 individuales propuestas, lo que parece apuntar a una buena implicación de los estudiantes con el entorno que les facilitaba la red 


\section{ANÁLISIS Y DISCUSIÓN}

Partiendo de una diferencia que pudiera parecer relevante, como es el hecho de proponer un análisis de la motivación dos materias que sobre el papel presentan muchas diferencias, se podrá observar, sin embargo, que el comportamiento frente a la red contiene muchas similitudes en ambos casos. De cualquier modo, los primeros elementos de la comparación se establecieron con la respuesta a un cuestionario de carácter anónimo, en dos fases, una primera al iniciarse el curso y una segunda al finalizar. El formulario fue idéntico en los dos casos y tuvo como objetivos los siguientes:

a) Detectar el grado de implicación de los estudiantes con las tecnologías.

b) Valorar sus propios conocimientos de informática.

c) Saber qué redes sociales conocían y en cuáles tenían cuenta.

d) Conocer su posición sobre el uso de las redes sociales en clase.

De acuerdo con sus respuestas, la receptividad que mostraron a la propuesta fue bastante alta, probablemente por el hecho de que, según ellos mismos revelaban, se trataba de su primera experiencia con una red social pedagógica, más allá del uso que confesaban de las redes conocidas, como Facebook, Tuenti u otras.

Al finalizar el curso, se repitió la encuesta variando preguntas que tenían relación con el nivel de satisfacción por el curso realizado. Entre las variaciones con la encuesta inicial se proponía catalogar el trabajo realizado en función de su utilidad, estableciendo una graduación que fue de "Útil" hasta "No aporta nada al aprendizaje". El resultado fue el que se presenta a continuación:

Tabla 2. Respuesta de la encuesta

\begin{tabular}{lcc}
\hline & Traducción 2008 & Français en Ligne \\
Útil & $6.6 \%$ & $46 \%$ \\
Muy útil & $73 \%$ & $46 \%$ \\
Motivadora & $86 \%$ & $61 \%$ \\
Complicada & 0 & 0 \\
Divertida & $40 \%$ & $30 \%$ \\
No aporta nada al aprendizaje & 0 & 0 \\
\hline
\end{tabular}

Como puede observarse, más allá de que la discordancia que representa el hecho de que para el grupo Traducción 2008 haya un 6.6\% que la considera "Útil” y un 73\% "Muy útil", se puede observar que la mayor atención se prestó al concepto Motivadora en ambos casos, si bien se observa una diferencia notable de un $25 \%$ en ambos grupos. Esta diferencia pudiera tener su explicación en el hecho de que, en el caso de Traducción 2008, la encuesta se pasó entre los meses de octubre de 2008 y enero de 2009. En aquel momento, de la constelación de redes sociales más utilizadas por los estudiantes, sobresalía con claridad Tuenti por encima de otras que con posterioridad desaparecieron o dejaron prácticamente de usarse. Tuenti era conocida 
por un $90 \%$ de los encuestados y en ella declaraban tener una cuenta. Sin embargo, el grupo Français en Ligne, que se estudió en el curso 2009-2010, ya presenta prácticamente el mismo número de usuarios de Tuenti que de Facebook y, cabe pensar, con un mayor desarrollo en su funcionamiento y actividad. Esta variable no ha podido medirse pero es natural que haya que atribuirle un valor, aunque se desconozca su relevancia.

Con relación a la utilidad que los estudiantes atribuyen a la red social pedagógica, los resultados son también bastante claros en sus líneas generales, si bien, como es natural, presenten peculiaridades. En este caso se les preguntó sobre diferentes opciones acerca de las ventajas que ofrece el uso de una red social en la clase. Estos fueron los resultados

Tabla 3. Respuesta acerca de las ventajas de la red

Estimula el trabajo cooperativo

Ayuda a mejorar las relaciones personales

Abre posibilidades de trabajo en equipo

Establece vínculos profesionales

No aporta gran cosa

Entorpece la clase

Valores
$86 \%$
$33.3 \%$
$93.3 \% 46 \%$
$66.6 \%$
00
0

Se observan diferencias que vienen dadas, en primer lugar, por la especificidad de los estudios. Así, parece bastante lógico que estudiantes de la licenciatura de Traducción consideren, de forma especialmente amplia, casi unánime, que la red abre posibilidades de trabajo en equipo, probablemente porque entienden que en su futura profesión de traductores estar conectado es un valor, verificable día a día, como se viene comprobando en la práctica profesional de la traducción. Este valor no parece excesivamente trascendente a futuros docentes de inglés y por ello el porcentaje desciende hasta un $46 \%$ siendo, aun así, significativo. Algo parecido ocurre con el ítem "Establece vínculos profesionales", donde observamos una diferencia significativa, de nuevo entre los futuros traductores, que parecen estar vislumbrando un futuro profesional, cosa que no ocurre con Français en Ligne.

En todo caso y, en lo que conforma el objetivo de este artículo, parece evidente que en ambos grupos el uso de la red fue lo suficientemente estimulante como para que se implicaran de forma general y decidida.

\section{CONCLUSIONES}

En el aprendizaje de los idiomas, el uso de las tecnologías y, más en concreto, las opciones que ofrece la web 2.0, adquiere día a día una dimensión diferente. Al tratarse de un aprendizaje instrumental, el uso de esta herramienta aporta elementos que no podían encontrarse en la enseñanza tradicional. Probablemente, el más esencial de todos ellos es el aprendizaje en contexto. 
Ningún otro dispositivo de las enseñanzas convencionales podía ofrecer esa posibilidad. En la actualidad, si un profesor desea proponer a sus alumnos una actividad que contenga elementos de tipo lingüístico asociados a otros de tipo cultural, todo ello en el contexto, por ejemplo, de la vida pública, no le resultará difícil encontrarlo sólo con acudir a Youtube, a una radio online, a un diario digital o a cualquier otro dispositivo en el que encontrará, por ejemplo, al presidente de Francia en un discurso ante una convención, a la actriz de moda en una entrevista de TV o al futbolista del momento en una entrevista digital de algún diario de gran tirada. Igualmente y, tomando en consideración que los jóvenes actuales han integrado la música en sus vidas con normalidad, una actividad de clase puede consistir en buscar la mejor canción francesa y votarla en un grupo de Facebook. Una vez votada, nadie se extrañará si el profesor propone buscar a su autor y ponerse en contacto con él para informarle y felicitarlo. El proceso se hace con toda la naturalidad de la que carecían los recursos utilizados tradicionalmente, que necesitaban impostar permanentemente las situaciones y necesitando de una especie de contrato latente entre profesor y estudiante para darle a la clase una apariencia de contexto.

Hoy eso ya no es necesario, pero tal vez lo más sorprendente sea que para nuestros estudiantes tampoco es extraordinaria la situación que se acaba de relatar. Sí lo es para los profesores de generaciones anteriores, y en esa fricción en la que nos encontramos tenemos, docentes y estudiantes, que buscar los puntos de encuentro. Entretanto, las redes sociales nos están proporcionando una herramienta de la que desconocemos prácticamente todas sus posibilidades. Intuimos que han de ser motivadoras, o muy motivadoras, para los alumnos, y las percepciones que alcanzamos así nos lo señalan

El estudio realizado está precisamente en esa línea y sus resultados apuntan a una alta aceptación de la red como elemento pedagógico, tal vez por su proximidad con Facebook, con Tuenti, con Twitter y otras, pero desconocemos cuál va a ser la evolución, incluso en el corto plazo. Por el momento solo podemos dar testimonio del alto grado de implicación de nuestros estudiantes, de su fácil integración en la clase, pero desconocemos casi todo sobre su futuro.

\section{REFERENCIAS}

Álvarez, A. (2009). Nuevas Tecnologías para la clase de Francés Lengua Extranjera: teoría y práctica. Madrid: Quiasmo Editorial.

Dans, E. (2010).Todo va a cambiar. Tecnología y evolución: adaptarse o desaparecer. Barcelona: Deusto (Grupo Planeta).

Artero Balaguer, N. (2011). La interacción como eje de aprendizaje en las redes sociales Educaweb.com. Recuperado el 27 de Julio de 2011, de http:/ / www.educaweb.com/noticia/2011/01/31/interaccion-como-eje-aprendizajeredes-sociales-14570.html 
Cabedo Gallén, Rosa (2011). Las redes sociales en el contexto formativo del siglo XXI" Educaweb.com. Recuperado el 27 de Mayo de 2011, de http://www.educaweb.com/noticia/2011/01/31/redes-sociales-contexto-formativosiglo-xxi-14569.html

\section{Alfredo Álvarez-Álvarez}

Doctor en Filología Francesa por la Universidad Autónoma de Madrid y profesor de la Universidad de Alcalá. Sus líneas de investigación pasan por la integración de las tecnologías en el aprendizaje del idioma y, más en concreto, del Francés. El resultado de sus investigaciones queda reflejado en más de 20 blogs, una decena de redes sociales, una web, además de colaboraciones en revistas y contribuciones a capítulos de libros. Entre sus libros, cabe señalar "Nuevas tecnologías para el aprendizaje del Francés: Teoría y práctica" (2009), Quiasmo Editorial, Madrid. 